\title{
3-D Diffusion Models for Chemically-Amplified Resists Using Massively Parallel Processors
}

\author{
E. Tomacruz, M. Zuniga, R. Guerrieri ${ }^{\dagger}$, A. Neureuther, and
}

A. Sangiovanni-Vicentelli

\author{
Department of Electrical Engineering \& Computer Sciences, University of California \\ Berkeley, CA 94720, USA \\ †Dipartimento di Elettronica, Informatica e Sistemistica, Università di Bologna \\ Viale Risorgimento 2, I-40136 Bologna, ITALY
}

\begin{abstract}
Three-dimensional concentration dependent diffusions and simultaneous chemical reactions in chemically-amplified photoresists are simulated. Fickian, a linearly increasing diffusivity and an exponentially increasing diffusivity due to free volume increase with T-BOC are considered. Two different grid to processor mappings are proposed in implementing the simulator on massively parallel processors using the Scharfetter and Gummel discretization method. Experimental and simulation results which support an exponential diffusion coefficient are illustrated.
\end{abstract}

\section{Introduction}

The lithographic process of transferring an ideal layout to a less than ideal resist profile on a wafer is an increasingly important consideration in IC design. The ability of the nonlinearity of the resist to produce vertical line-edge profiles from low quality images in the presence of sharp vertical standing waves is an important technological improvement which has extended the working resolution of optical lithography. The improvement is especially significant for chemically-amplified resist systems which have both nonlinear chemical reaction kinetics and simultaneous concentration dependent diffusion. The level of complexity in modeling these reaction and diffusion effects is similar to that involved in modeling impurity concentration and point defect dependent diffusion in silicon. The use of high post-bake temperatures is preferred as resist sensitivity improves. However, it results in increased diffusion which must be examined in three dimensions. The nonlinearity of the reactions and the dependence of the diffusion on the local concentration can lead to improved performance. Understanding and balancing these mechanisms is the key goal in designing production worthy resists.

This research project models the 3D movement and reaction of species in the postexposure bake of chemically-amplified resist systems. Both weak and strong dependencies of diffusion on species concentration are considered. Combinations of nonlinear reaction kinetics and concentration dependent diffusion scenarios are being considered for both acid-hardening (negative) resist and deprotection reaction (positive) resist. A bidirectional link to the SAMPLE-3D lithography simulator is established for facilitating studies of 3D effects. 


\section{Computational Models}

A general model for two interacting and one diffusing species summarized by the equations below is used [1].

$$
\begin{gathered}
\frac{\partial C_{1}}{\partial t}=k_{1}\left(1-C_{1}\right) C_{2}^{1.42}, \frac{\partial C_{2}}{\partial t}=\nabla \cdot\left(D_{2} \nabla C_{2}\right)+k_{2} C_{2}, \frac{\partial}{\partial z} C_{2}(x, y, z)=0^{\prime}{ }_{z=0, T}, \\
C_{1}(x, y, z)=0^{\prime}{ }_{t=0}, C_{2}(x, y, z)=C_{s s}{ }_{z=0}
\end{gathered}
$$

where $C_{1}$ is the concentration of activated sites, $C_{2}$ is the concentration of acid, $k_{1}$ is the reaction rate coefficient, and $k_{2}$ is the rate coefficient for the acid loss reaction. $D_{2}$ is the diffusion coefficient which may be dependent on $C_{l}$. The terms with $k$ coefficients model a class of resist materials that rely on the acid catalyzed cross-linking of the resin matrix during post-exposure bake. The third equation, where $T$ is resist thickness, specifies that no net flow of acid occurs across the boundaries. In a silylation process, silicon-containing compounds diffuse into the resist from an outside source. This is modeled by the fifth equation which forces the concentration of the species to be the constant $C_{s s}$ at the resist surface.

The diffusion is not well understood and might be due to the nonlinear dependence of the diffusion rate on 1) the acid concentration itself (constant)[2], (2) the presence of deprotection sites which provide additional stepping stones (linear model), or (3) the increase in free volume with the deprotection reaction which creates a very rapid increase in diffusion pathways (exponential model)[3]. To explore the possibility of these various classes of acid concentration and material state dependent diffusion a general purpose 3-D reaction-diffusion simulator was written. For each of the three classes of increasingly higher nonlinearity an appropriate algorithm was chosen.

$$
D_{2}=\alpha, D_{2}=\gamma+\beta C_{1}, D_{2}=\lambda \exp \left(\omega C_{1}\right)
$$

where $\alpha, \gamma, \beta, \lambda$, and $\omega$ are constant parameters.

\section{Discretization and Computational Steps}

Since the exponential diffusion coefficient is a highly non-linear function of the variables, the standard difference discretization is not suitable for the task unless the grid spacings are made very small. To attain a more stable discretization, we adopt a technique proposed by Scharfetter and Gummel [4] which is now widely used for the discretization of the semiconductor device equations. The same approach is used for the discretization of the linear diffusion coefficient. Taking the limits of the linear diffusion coefficient discretization, the discretization for the constant diffusion coefficient is obtained.

Given that the flow of species $C_{2}$, is described as

$$
J=\alpha \nabla C_{2}, J=\left(\gamma+\beta C_{1}\right) \nabla C_{2}, J=\lambda \exp \left(\omega C_{1}\right) \nabla C_{2}
$$

where $C_{1}$ is the other species interacting with $C_{2}$, two simplifying assumptions are made. First, $C_{1}$ is linearly discretized. Second, the flow of $C_{2}$ is constant between grid nodes in a one-dimensional grid. Using techniques used by Scharfetter and Gummel, the constant value of the flux can be extracted.

$$
\begin{gathered}
J=\alpha \frac{C_{2(i)}-C_{2(1-1)}}{x_{i}-x_{i-1}}, J=\frac{\beta\left(C_{1(i)}-C_{1(i-1)}\right)}{\ln \left(\frac{\gamma+\beta C_{1(i)}}{\gamma+\beta C_{1(i-1)}}\right)} \frac{C_{2(i)}-C_{2(i-1)}}{x_{i}-x_{i-1}}, \\
J=\lambda \frac{-\omega\left(C_{1(i)}-C_{1(i-1)}\right)}{\left(\exp \left(-\omega C_{1(i)}\right)-\exp \left(-\omega C_{1(i-1)}\right)\right)} \frac{C_{2(i)}-C_{2(i-1)}}{x_{i}-x_{i-1}}
\end{gathered}
$$


Rectangular grids with nonuniformly spaced lines are used for the discretization. The nonlinear equations are solved using the Newton-Raphson method and the second order trapezoidal method is used for numerical integration. The Conjugate Gradient Squared (CGS) iterative algorithm is used to solve the unsymmetric sparse matrix. Incomplete LU decomposition with the partitioned natural ordering [5] is used as a preconditioner for the linear solver. At each time point, a nonlinear system of equations is solved using the Newton-Raphson method. For each Newton-Raphson iteration, a linear system of equations is solved. These computational steps are CPU time expensive for 3-D simulations but are expected to give convergent results that are accurate even for non-constant diffusion dependencies. Since mesh structures with over 100,000 nodes are not uncommon, supercomputing machines are necessary. The Connection Machine $2(\mathrm{CM}-2)$ and the Connection Machine $5(\mathrm{CM}-5)$ computer architectures used for this study offer the computation power needed. Hence, massively parallel processors (MPPs) make 3-D simulations of reaction kinetics and diffusion in advanced resist processes practical.

\section{Algorithmic Mapping}

For the CM-2, each grid point in the mesh structure is mapped into a corresponding processor. Thus each processor stores the local values of $C_{1}$ and $C_{2}$, and the two rows of the matrix of the corresponding grid node. Using this allocation, the grid fits naturally on the organization of the machine and, at the same time, variables having a strong coupling due to spatial adjacency are tightly clustered. A reduction in the size of the matrix can be obtained by replacing $C_{1}$ with a function of $C_{2}$. With $256 \mathrm{k}$ bits of memory for each processor, a virtual processor ratio of 64 is obtained. Hence, an $8 \mathrm{k} \mathrm{CM}-2$ with $256 \mathrm{k}$ bits of memory for each processor would allow a user to simulate a $512 \mathrm{k}$ mesh structure.

The CM-5 mesh structure is divided into rectangular blocks each called a subdomain [6]. Each subdomain is mapped to a processor and the dimensions of the subdomains are equal in order to form cube partitions. This minimizes the total surface area which in turn minimizes the data length of communications between processors. A simple row ordering is used to map the subdomains to the CM-5 processors since the fat tree connections allow minimal penalty for communications between arbitrary processors [7].

\section{Experimental and Simulation Results}

Preliminary experimental data illustrated in Figure 1 show evidence of diffusion effects in I.B.M. APEX-E resist profiles. A Fisher F-test shows that a linewidth loss linear in time best describes Figure 1 since a linear model provides smaller sums of residuals compared to a quadratic model [8]. Also, the resist tends to have very vertical sidewalls regardless of exposure or bake time which would suggest a vertical front is propagating laterally through the resist. Figure 2 shows simulation results for the three proposed diffusion models. $\beta$ is $1.0 \mathrm{e}-18$ for linear $1, \beta$ is $1.0 \mathrm{e}-17$ for linear $2, \lambda$ is $0.3 \mathrm{e}-19$ for $\exp 1, \lambda$ is $1.0 \mathrm{e}-19$ for $\exp 2$, and $\lambda$ is $1.0 \mathrm{e}-18$ for $\exp 3$. $\alpha$ is $1.0 \mathrm{e}-18, \gamma$ is $1.0 \mathrm{e}-18$, and $\omega$ is 5.0 for all simulations. Only the exponential model could show a linear trend and, as illustrated in Figure 3, it is the only model observed to have a vertical front propagating through the resist.

Current simulation data also show high average of acid concentration associated with the fraction of a standing wave cycle near the resist surface. This high average forms a foot and top-lip on the resist profile which are usually not seen in experimental results. Only the exponential diffusion coefficient has sufficient vertical acid movement to nearly eliminate foot and top-lip formations. More experimental results are presented in [8]. 


\section{References}

[1] R. Ferguson, Ph.D. Thesis, University of California, Berkeiey, May 1991.

[2] T. Yoshimura et.al., J. Vac. Sci. Teclınol. B, Vol. 10, No. 6, 1992.

[3] W. Hinsberg et.al., SPIE Proceedings, Vol. 1925, 1993.

[4] D.L. Scharfetter, H.K. Gummel, IEEE Trans. on Electron Devices, Vol. ED-16, pp. 66-77, 1969.

[5] D. Webber et.al., IEEE Trans. on CAD, Vol. 10, pp. 1201-1209, 1991.

[6] E. Tomacruz et.al., International Workshop on VLSI Process and Device Modeling, pp. 20-21, 1993.

[7] Z. Bozkus et.al., IEEE 4th Symp on the Frontiers of Massively Parallel Computation, pp. 100-107, 1992.

[8] M. Zuniga et.al., Submitted to Elec., Ion, and Photon Beams Conference, San Diego, CA, USA, 1993.

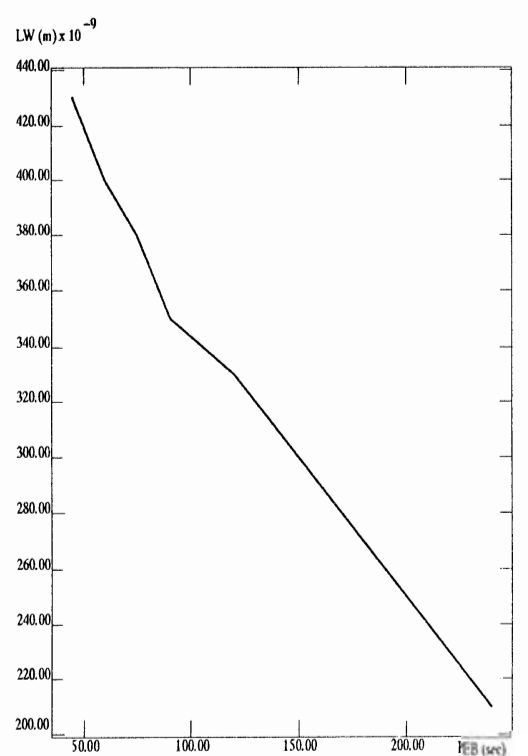

Figure 1: Experimental Results

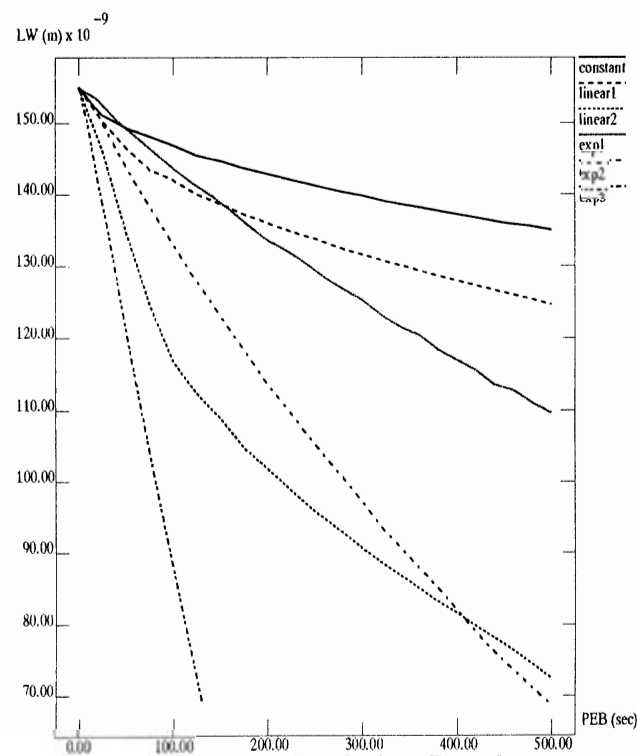

Figure 2: Simulation Results

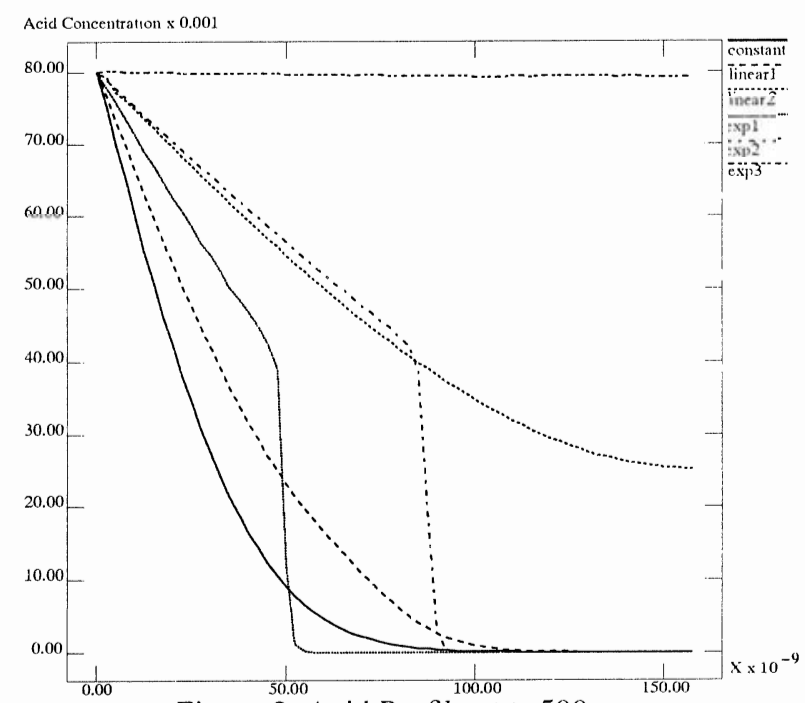

Figure 3: Acid Profile at $t=500 \mathrm{sec}$ 